\title{
La colaboración y la Biblioteca Cochrane en Urología
}

\author{
C. Tello Royloa*, G. Urrutia Cuchí** \\ *Grupo de Trabajo en Urología Basada en Pruebas de la Asociación Murciana de Urología. \\ ${ }^{* *}$ Centro Cochrane Iberoamericano. \\ Actas Urol Esp 2005: 29 (3): 244-251
}

\section{RESUMEN}

LA COLABORACIÓN Y LA BIBLIOTECA COCHRANE EN UROLOGÍA

La Colaboración Cochrane es una organización internacional, sin fines de lucro, que busca ayudar a quienes necesitan tomar decisiones bien informadas en atención sanitaria. Su principal objetivo es: Preparar, actualizar, promover y facilitar el acceso a las revisiones sistemáticas sobre las intervenciones de la atención sanitaria. Su principal producto es la Biblioteca Cochrane (The Cochrane Library), una publicación electrónica trimestral en formato electrónico. Se describen los principios y la estructura de la Colaboración Cochrane y la estructura y contenido de la Biblioteca Cochrane. Se expone la importancia de las revisiones sistemáticas y de los meta-análisis como fuentes de información para los clínicos, destacando las aportaciones de la Colaboración Cochrane en la especialidad de urología.

Palabras clave: Urología. Medicina Basada en la Evidencia. Bases de datos. Bibliotecas, médicas.

\section{ABSTRACT}

THE COCHRANE COLLABORATION AND THE COCHRANE LIBRARY IN UROLOGY

The Cochrane Collaboration is an international, non-profit making organisation which aims to help people make well-informed health care decisions. The main objective is to prepare, update, promote and facilitate access to systematic reviews on health care interventions. The main product is The Cochrane Library, a quarterly electronic publication. The principles and structure of the Cochrane Collaboration and the structure and content of the Cochrane Library will be described. The importance of systematic reviews and meta-analyses as sources of information for clinicians will be presented, with special emphasis on the contributions of the Cochrane Collaboration to the speciality of urology.

Keywords: Urology. Evidence-based Medicine. Databases. Libraries, Medical.

$\mathrm{E}$ 1979 el epidemiólogo inglés Archie Cochrane advirtió a la comunidad médica sobre la conveniencia de elaborar un resumen crítico y actualizado de todos los ensayos clínicos relevantes, organizados por especialidades o subespecialidades ${ }^{1}$.

$\mathrm{El}$ enorme volumen y la variable calidad de la literatura científica, hace que estudios de impecable metodología y gran relevancia clínica queden oscurecidos entre otros muchos intrascendentes o de dudoso rigor científico. Y en cualquier caso, la proliferación de publicaciones, hace imposible a los clínicos el conocimiento directo de la mayor parte de los estudios publicados, aún en su propia especialidad o "superespecialidad". Se hace por ello necesaria la síntesis de la literatura primaria en resúmenes o revisiones secundarias que seleccionen y trasmitan las mejores pruebas o "evidencias" de la investigación clínica. Pero la investigación secundaria, como proceso de búsqueda, valoración crítica y síntesis de la información debe ser exhaustiva, rigurosa y reproducible. 
Además, pequeños ensayos clínicos publicados, o que nunca llegaron a publicarse, apuntan con frecuencia tendencias sobre la posible utilidad o inutilidad de un tratamiento o una técnica diagnóstica, sin alcanzar resultados concluyentes por la escasez de la muestra. Frases como: "Son necesarios más estudios..." se leen a menudo en las conclusiones de muchos trabajos. En el otro extremo, los "mega-ensayos" que permiten obtener resultados fiables y precisos son demasiado costosos y en ocasiones irrealizables. Las revisiones sistemáticas, como herramientas de sintesis de la información, y los meta-análisis como instrumentos que resumen de forma cuantitativa los resultados de muchos ensayos clinicos dispersos, permiten en ocasiones alcanzar conclusiones relevantes sin necesidad de realizar costosos "mega-ensayos".

$\mathrm{El}$ rápido crecimiento del conocimiento científico y la incesante aparición de nuevas pruebas, hace que incluso las mejores revisiones queden desfasadas a los pocos años o incluso meses de su publicación. La actualización de las revisiones es, por tanto, un elemento esencial.

Con este objetivo de preparar, actualizar y difundir revisiones sistemáticas sobre las intervenciones sanitarias, nació en 1993 la Colaboración Cochrane.

El objetivo de este trabajo es dar a conocer a la comunidad urológica la organización y funcionamiento de la Colaboración Cochrane, el fruto de su trabajo, que se recoge en la Biblioteca Cochrane (The Cochrane Library), y sus aportaciones al campo de la urología.

\section{LA COLABORACIÓN COCHRANE}

La Colaboración Cochrane es una organización internacional, sin fines de lucro, que busca ayudar a quienes necesitan tomar decisiones bien informadas en atención sanitaria. Su principal objetivo es: Preparar, actualizar, promover y facilitar el acceso a las revisiones sistemáticas sobre las intervenciones de la atención sanitaria.

\section{PRINCIPIOS DE LA COLABRACIÓN COCHRANE}

La actividad de la Colaboración Cochrane se basa en los siguientes de principios filosóficos:

- Basar su desarrollo en el entusiasmo de los individuos, involucrando y dando apoyo a personas con diferente formación y experiencia diversa.
- Evitar la duplicación, mediante una buena gestión y coordinación para que los esfuerzos sean eficientes.

- Minimizar los sesgos, gracias a una variedad de estrategias, tales como el rigor científico, una amplia participación y el reconocimiento de posibles conflictos de interés.

- Actualización permanente, asumiendo el compromiso de asegurar que las revisiones Cochrane identificarán e incorporarán la nueva evidencia que vaya apareciendo.

- Priorización de la relevancia, promoviendo la evaluación de las intervenciones sanitarias a partir de resultados que sean importantes para las personas que deben tomar decisiones.

- Promover el acceso, mediante una amplia diseminación de los productos de la Colaboración, aprovechando las alianzas estratégicas y asegurando una política de precios, un contenido y unos medios adecuados para cubrir las necesidades de los usuarios en todo el mundo.

- Asegurar la calidad, permaneciendo abiertos y sensibles a las críticas, aplicando los avances metodológicos y desarrollando sistemas para la mejora continua de la calidad.

- Organizar y gestionar una estructura eficiente y transparente, y minimizar la jerga burocrática introducida en las publicaciones.

- Colaborar gracias al impulso de una buena comunicación interna y externa, de unas decisiones transparentes y del trabajo en equipo.

- Continuidad, asegurando que la responsabilidad relacionada con los revisores, los procesos editoriales y otras funciones clave se mantienen y renuevan ${ }^{2}$.

\section{EL LOGOTIPO DE LA COLABORACIÓN COCHRANE}

El logotipo de la Colaboración Cochrane es un diagrama que refleja el resultado del meta-análisis de una revisión sistemática de siete ensayos clínicos sobre el efecto de una pauta corta de tratamiento con corticoesteroides en mujeres gestantes con amenaza de parto prematuro, en la mortalidad neonatal por inmadurez fetal. 
El primero de estos ensayos se publicó en el año 1972. El meta-análisis de los siete ensayos clínicos, realizado en 1991, indica que este tratamiento reduce entre un 30 y un 50 \% la probabilidad de morir de estos recién nacidos, a causa de las complicaciones derivadas de la inmadurez fetal.

Dado que no se publicó ninguna revisión sistemática de estos ensayos hasta 1989, la mayoría de los obstetras no conocían que el tratamiento era tan efectivo y, probablemente, decenas de miles de recién nacidos prematuros padecieron y murieron como consecuencia de este desconocimiento $^{3}$ (Fig. 1).

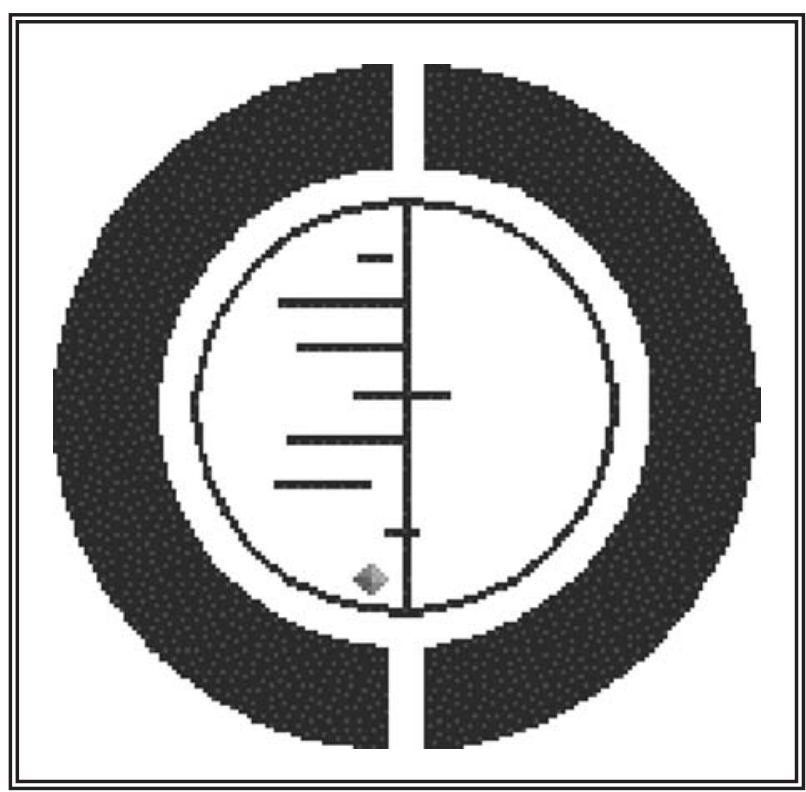

FIGURA 1. Logotipo de la Colaboración Cochane.

\section{ESTRUCTURA DE LA COLABORACIÓN COCHRANE}

En la actualidad, más de 5000 personas de más de 50 países están colaborando activamente con la Colaboración Cochrane, que ha recibido además el apoyo por parte de más de 650 organizaciones de todo el mundo, incluyendo proveedores sanitarios, agencias de investigación, departamentos de salud, organizaciones internacionales, industria y universidades ${ }^{3}$.

La Colaboración Cochrane se estructura en varios niveles o entidades, cada una de los cuales tiene responsabilidades $\mathrm{y}$ funciones específicas.
Estas entidades son: Los Grupos Colaboradores de Revisión (Collaborative Review Group), los Grupos de Metodología (Methods Working Groups), los Ámbitos (Fields), la Red de Consumidores (Consumer Network), los Centros, el Comité Directivo y el Secretariado.

\section{Los Grupos Colaboradores de Revisión.}

Los Grupos Colaboradores de Revisión (CRG, acrónimo de "Collaborative Review Group") se encargan de preparar y actualizar las revisiones sistemáticas. Son grupos de carácter internacional, multidisciplinario, integrados por investigadores, clínicos, profesionales de la salud, usuarios de los servicios sanitarios, etc., que se han reunido porque comparten el interés por un problema específico de salud como: las "enfermedades de la próstata y el cáncer urológico", la "incontinencia urinaria”, las "enfermedades renales", etc. En la Tabla 1 aparecen recogidos todos los Grupos de Revisores existentes en la actualidad. (2004).

Las actividades de los revisores integrados en un CRG está facilitada por un equipo editorial, designado por el mismo Grupo, que coordina, organiza, supervisa y asesora las actividades del mismo.

\section{Los Grupos de Métodos (Methods Working Groups)}

Los Grupos de Métodos se han establecido para desarrollar los métodos y aconsejar a la Colaboración sobre cómo mejorar la validez y precisión de las revisiones sistemáticas. Por ejemplo, el Grupo de Trabajo sobre Metodología Estadística (Statistical Methods Working Group) evalúa las alternativas existentes para manejar los diferentes tipos de datos en la síntesis estadística, y el Grupo de Trabajo sobre Aplicabilidad y Recomendaciones (Applicability and Recommendations Methods Working Group) explora cuestiones importantes acerca de la obtención de conclusiones sobre las implicaciones para la práctica, basándose en los resultados de las revisiones.

\section{Los Ámbitos (Fields)}

Los Ámbitos se ocupan de otras dimensiones sanitarias más allá de los problemas de salud tales como: la administración de la atención sanitaria (p. ej.: atención primaria), el tipo de consumidor (p. ej.: tercera edad), o tipo de intervención (p. ej.: vacunación). 
Tabla 1

Lista de los Grupos Cochrane de Revisión (en 2004)

Grupo Cochrane de Infecciones Respiratorias Agudas

Grupo Cochrane de Vias Respiratorias

Grupo Cochrane de Anestesia

Grupo Cochrane de Trastornos de la Espalda

Grupo Cochrane de Cáncer de Mama

Grupo Cochrane de Cáncer Colorrectal

Grupo Cochrane de Consumidores y Comunicación

Grupo Cochrane de Fibrosis Quística y Enfermedades Genéticas

Grupo Cochrane de Demencia y Trastornos Cognitivos

Grupo Cochrane de Depresión, Ansiedad y Neurosis

Grupo Cochrane de Problemas de Desarrollo, Psicosociales y de Aprendizaje

Grupo Cochrane de Drogas y Alcohol

Grupo Cochrane de Enfermedades de Oído, Nariz y Garganta

Grupo Cochrane para una Práctica y Organización Sanitaria Efectivas

Grupo Cochrane de Epilepsia

Grupo Cochrane de Ojos y Visión

Grupo Cochrane de Regulación de la Fertilidad

Grupo Cochrane de Cáncer Ginecológico

Grupo Cochrane de Neoplasias Hematológicas

Grupo Cochrane de Enfermedades del Corazón

Grupo Cochrane de Enfermedades Hepato-biliares

Grupo Cochrane de Sida/VIH

Grupo Cochrane de Hipertensión

\section{Grupo Cochrane de Incontinencia}

Grupo Cochrane de Enfermedades Infecciosas

Grupo Cochrane de Enfermedad Inflamatoria Intestinal

Grupo Cochrane de Lesiones

Grupo Cochrane de Cáncer de Pulmón

Grupo Cochrane de Trastornos Menstruales y Subfertilidad

Grupo Cochrane de Enfermedades metabólicas y endocrinológicas

Grupo Cochrane de Metodología

Grupo Cochrane de Trastornos del Movimiento

Grupo Cochrane de Esclerosis Múltiple

Grupo Cochrane de Lesiones Musculosqueléticas

Grupo Cochrane de Enfermedades Musculosqueléticas

Grupo Cochrane de Neonatología

Grupo Cochrane de Enfermedades Neuromusculares

Grupo Cochrane de Salud Oral

Grupo Cochrane de Dolor, Apoyo y Curas Paliativas

Grupo Cochrane de Enfermedades Vasculares Periféricas

Grupo Cochrane de Embarazo y Parto

Grupo Cochrane de Enfermedades de la Próstata y Cáncer Urológico

\section{Grupo Cochrane de Enfermedades Renales}

Grupo Cochrane de Esquizofrenia

Grupo Cochrane de Enfermedades de Transmisión Sexual

Grupo Cochrane de Piel

Grupo Cochrane de Embolia

Grupo Cochrane de Adicción al Tabaco

Grupo Cochrane de Enfermedades Gástricas, del Intestino Delgado y Pancreáticas

Grupo Cochrane de Heridas

En negrita los Grupos más directamente relacionados con patología urológica.
Las personas vinculadas a los Ámbitos buscan fuentes especializadas de estudios relevantes, ayudan a asegurar que las prioridades y perspectivas en sus esferas de interés se vean reflejadas en los Grupos Colaboradores de Revisión, compilan bases de datos especializadas, coordinan actividades con agencias clave de fuera de la Colaboración Cochrane y hacen observaciones sobre las revisiones sistemáticas relacionadas con sus áreas respectivas.

\section{Red de Consumidores (Consumer Network)}

La Red Cochrane de Consumidores (Cochrane Consumer Network) proporciona información y se abre como foro para vincular entre sí a los consumidores participantes en la Colaboración y es un puente entre los grupos de consumidores y usuarios de los sistemas de salud alrededor del mundo.

\section{Centros}

El trabajo de los Grupos Colaboradores de Revisión, de los Grupos de Metodología, de los Ámbitos y de la Red de Consumidores está apoyado por la tarea de los Centros Cochrane distribuidos por todo el mundo. Comparten la responsabilidad de ayudar a coordinar y dar respaldo a los miembros de la Colaboración en áreas tales como formación, así como promover objetivos de la Colaboración en su ámbito geográfico.

El Centro Cochrane Iberoamericano, ubicado en el Hospital de la Santa Creu i Sant Pau de Barcelona es uno de los 12 Centros Cochrane internacionales actualmente existentes y está constituido por una red de Centros Coordinadores Nacionales ubicados en diversos países del ámbito iberoamericano como son España, Argentina, Chile, Colombia, Cuba, México, Costa Rica y Venezuela. Existen también grupos de trabajo constituidos en Portugal y Uruguay. 
Comité Directivo y Secretariado

El Comité Directivo está integrado por 14 miembros que se reúnen dos veces al año. En medio de estos dos encuentros, el Comité Directivo mantiene reuniones periódicas entre sus diferentes grupos de trabajo por teleconferencia. Sus decisiones están guiadas por metas y objetivos establecidos en el Plan Estratégico de la Colaboración (Collaboration's Strategic Plan).

El Comité Directivo y sus varios grupos de trabajo están apoyados por una pequeña secretaría actualmente localizada en el Reino Unido.

\section{LA BIBLIOTECA COCHRANE}

La Biblioteca Cochrane (The Cochrane Library) es el principal producto de la Colaboración Cochrane. Es una publicación electrónica que se actualizada cada tres meses. Se distribuye mediante suscripción anual en $\mathrm{CD}$ o a través de Internet. En algunos países existe un acceso universal gratuito, ya sea por subscripciones realizadas por los gobiernos (Irlanda, Reino Unido, Australia, Nueva Zelanda, Finlandia, Noruega, Dinamarca, etc.) o por acuerdos internacionales de acceso gratuito a las fuentes de información en los países en vías de desarrollo. La Versión en español, La Cochrane Library Plus, está disponible en formato "on-line" también de forma gratuita desde octubre de 2003 gracias al acuerdo suscrito por el ministerio de Sanidad y Consumo (acceso a través de la web www.update-software.com/clibplus/clibplus.htm).

La Biblioteca Cochrane incluye varias bases de datos, entre las que destacan la Base de datos de revisiones sistemáticas y el registro de ensayos controlados.

La Base de Datos Cochrane de Revisiones Sistemáticas - BDCRS (The Cochrane Database of Systematic Reviews-CDSR)

Contiene dos tipos de documentos: Las Revisiones y los Protocolos Cochrane. Las Revisiones Cochrane son revisiones sistemáticas, elaboradas y actualizadas por los distintos Grupos de Revisión. Los Protocolos aportan información sobre las revisiones que están en proceso de realización. Incluye un sistema de comentarios y críticas que permite a los usuarios ayudar a mejorar la calidad de las Revisiones.

Actualmente (Número 4 de 2004), la BDCRS contiene 2.170 revisiones terminadas y 1.500 protocolos.
El Registro Central Cochrane de Ensayos Controlados. (The Cochrane Central Register of Controlled Trials - CENTRAL).

Actualmente, con 427.807 referencias, constituye la mayor base de datos disponible sobre ensayos clínicos controlados. Éstos son identificados gracias a un esfuerzo coordinado a nivel mundial que incluye la búsqueda manual en más de 1700 revistas biomédicas en diversas lenguas, búsquedas electrónicas en bases de datos y esfuerzos conjuntos con la industria farmacéutica y otros colectivos a fin de asegurar que todos los ensayos clínicos identificados sean accesibles, a fin de evitar el sesgo de publicación o de selección que, en última instancia, puedan conducir a estimaciones sesgadas de los efectos de las intervenciones sanitarias ${ }^{3}$.

Además, la Biblioteca Cochrane incluye otras bases de datos y documentos como:

La Base de Datos de Resúmenes de Revisiones de Efectividad (The Database of Abstracts of Reviews of Effectiveness - DARE). Contiene evaluaciones criticas y resúmenes estructurados de otras revisiones sistemáticas, no Cochrane, según unos criterios de calidad explícitos (actualmente 4918 documentos). Esta base de datos está producida y actualizada por el Centro de Revisiones y Divulgación del Sistema de Salud Británico (NHS Centre for Reviews and Dissemination en la Universidad de York).

La Base de Datos Cochrane de Revisiones de Metodología. (Cochrane Database of Methodology Reviews - CDMR). Contiene las revisiones sistemáticas a texto completo y los protocolos (proyectos de revisiones) de estudios sobre metodología.

El Registro Cochrane de Metodología. (The Cochrane Methodology Register - CMR). Contiene referencias bibliográficas de artículos y libros sobre la ciencia de revisar la investigación.

La Base de Datos de Evaluación Económica de la NHS. (The NHS Economic Evaluation DatabaseNHS EED). Incluye resúmenes estructurados de artículos sobre evaluaciones económicas y está producida por el NHS Centre for Reviews and Dissemination.

La Base de Datos de Evaluación de Tecnologias Sanitarias. (Health Technology Assessment Database-HTA). Contiene información sobre evaluación de tecnologías sanitarias y está producida también por el NHS Centre for Reviews and Dissemination. 
El Manual Cochrane ${ }^{4}$. Metodología para la realización de revisiones sistemáticas y Un Glosario de términos.

Y los datos de contacto con los Grupos de Revisores y otras entidades de la Colaboración Cochrane.

\section{REVISIONES SISTEMÁTICAS Y META-ANÁLISIS}

Las revisiones bibliográficas o revisiones de la literatura científica son estudios, muy habituales y necesarios, que pretenden sintetizar y actualizar los conocimientos sobre un tema determinado. Las llamadas revisiones tradicionales o narrativas, aunque exhaustivas a veces, suelen carecer de una sistemática en la búsqueda, selección, evaluación y análisis de los estudios, con el consiguiente riesgo de que las conclusiones resulten sesgadas. Frente a estas revisiones tradicionales, ampliamente utilizadas y difundidas, está surgiendo en los últimos años el interés por otras herramientas de síntesis de la información como son: las revisiones sistemáticas y los meta-análisis.

Las revisiones sistemáticas son estudios de investigación secundaria, sobre cuestiones claramente formuladas, que resumen y sintetizan los resultados de múltiples investigaciones primarias, utilizando una metodología de identificación, selección, evaluación crítica y síntesis de la información, que limita los sesgos y los errores aleatorios.

En ese intento de reducir la subjetividad y los sesgos, la metodología de las revisiones sistemáticas comienza con la elaboración de un protocolo o plan de trabajo en el que se establece de forma explícita y a priori: los objetivos concretos de la revisión (pregunta clínica), las fuentes de información que se van a consultar, las estrategias de búsqueda que se van a utilizar y los criterios para la selección de los estudios. Los estudios obtenidos se analizan críticamente, con frecuencia por varios revisores independientes, para evaluar su validez y decidir su inclusión o exclusión. Los datos de los trabajos incluidos se tabulan mediante un formulario de recogida de datos específico previamente diseñado. El análisis de los datos obtenidos y la presentación de los resultados incluye una síntesis cualitativa o descriptiva de los resultados y, cuando es posible, la integración numérica de los mismos mediante la herramienta estadística del meta-análisis.

\section{REVISIONES SISTEMÁTICAS DE LA COLABORACION COCHRANE}

Las revisiones sistemáticas elaboradas por la Colaboración Cochrane comparten, además, algunas características que las hacen especialmente útiles: su carácter dinámico, su estructura y el apoyo metodológico que reciben sus autores de la Colaboración Cochrane.

Los revisores de la Colaboración Cochrane tienen un compromiso de mantener actualizadas sus revisiones, incluyendo las nuevas "evidencias" que van surgiendo. Cada trimestre se añaden a la Biblioteca Cochrane, no sólo las nuevas revisiones, sino también las actualizaciones de las revisiones existentes.

Una de las características más interesantes de las revisiones Cochrane es la uniformidad de su formato. Todas las revisiones se realizan con la ayuda de un programa informático llamado Review Manager (RevMan) y comparten un esquema común, que facilita su lectura y permite localizar rápidamente la información. En la Tabla 2 se presenta el esquema las revisiones Cochrane.

\section{LA COLABORACION COCHRANE EN UROLOGÍA}

La organización de los Grupos de Revisión de la Colaboración Cochrane (50 actualmente) se ha realizado más en función de patologías o temas de interés, que siguiendo el esquema tradicional de las especialidades médicas. Actualmente existen al menos cuatro grupos que trabajan en patologías y trastornos de interés para el urólogo:

El Grupo Cochrane de Enfermedades de la Próstata y Cáncer Urológico. Es el grupo más específico sobre patología urológica. Ha realizado revisiones sobre el efecto de distintos tratamientos de la hiperplasia prostática benigna como el Cermilton, los beta-sitosteroles, el Pygeum africanum, la Serenoa repens, la Tamsulosina o la Terazosina y están en fase de elaboración (protocolos) otras revisiones sobre el efecto de la alfuzosina y la doxazosina. Ha publicado también revisiones sobre el efecto del bloqueo androgénico en el cáncer de próstata, sobre el empleo de la inmunoterapia en el cáncer renal avanzado, sobre la BCG en los tumores vesicales superficiales, sobre la cistectomía radical versus radiotera- 
Tabla 2

Formato de una revisión Cochrane.

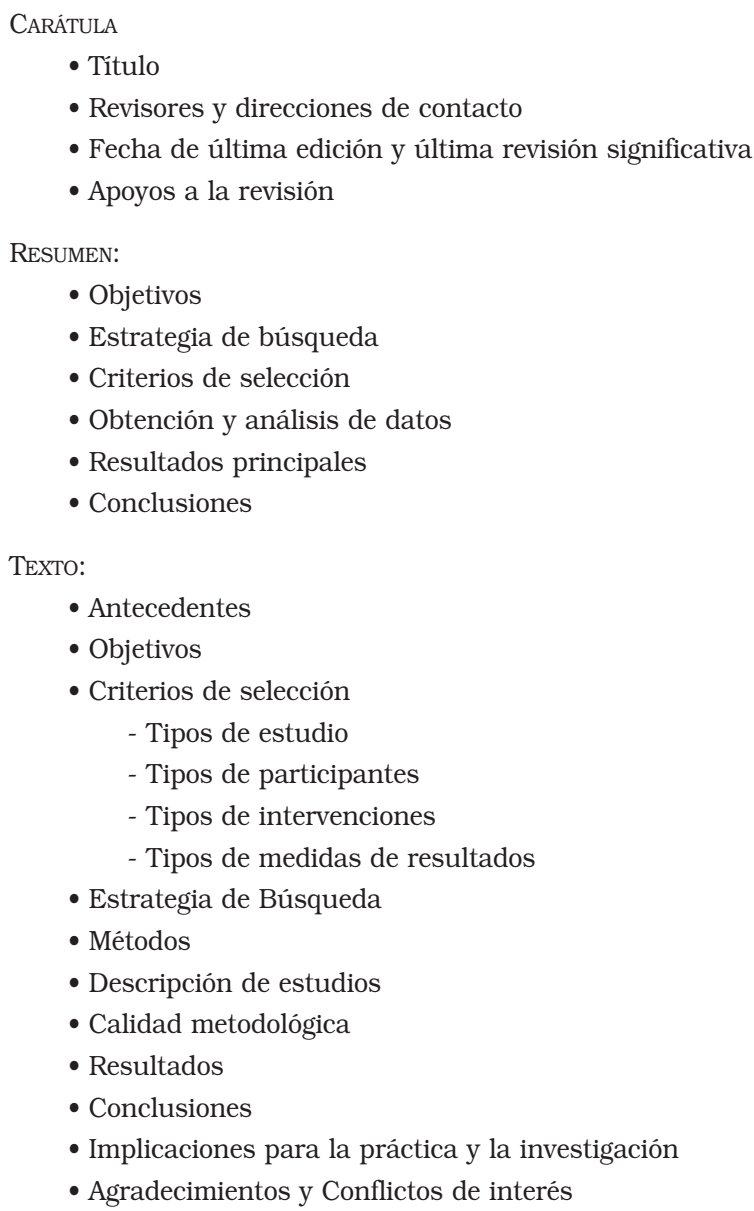

- Implicaciones para la práctica y la investigación

- Agradecimientos y Conflictos de interés

TABLAS Y FIGURAS

REFERENCIAS: REFERENCIAS DE LOS ESTUDIOS (INCLUIDOS Y EXCLUIDOS) - Otras referencias (adicionales...)

pia y sobre el efecto de la quimioterapia neoadyuvante en los tumores vesicales infiltrantes, y sobre el tratamiento de las prostatitis ${ }^{5}$.

El Grupo Cochrane de Incontinencia. Originalmente llamado Grupo Cochrane para la incontinencia urinaria y fecal, fue registrado en la Colaboración en 1996. El centro de atención de este Grupo son las investigaciones diseñadas para prevenir o tratar la incontinencia o ayudar a su rehabilitación. Los problemas cubiertos incluyen la incontinencia urinaria y fecal, las fistulas cutáneointestinales y vésico-intestinales, la enuresis, la encopresis, el uso de catéteres urinarios y las infecciones relacionadas con su uso (no el resto de infecciones urinarias que son abordadas por el Grupo de enfermedades renales), los trastornos miccionales de origen neurológico, los prolapsos rectales y vaginales. Ha realizado revisiones sobre distintos tratamientos y técnicas quirúrgicas en la incontinencia urinaria femenina (colporrafia anterior, suspensión del cuello vesical con agujas, colposuspensión laparoscópica, sling suburetral, inyecciones periuretrales, ejercicios del suelo pélvico estrógenos, conos vaginales), sobre los distintos tratamientos de la enuresis (Desmopresina, antidepresivos tricíclicos, otros fármacos, terapias de comportamiento o sistemas de alarma) ${ }^{6}$.

El Grupo Cochrane de Enfermedades Renales. Centrado sobre todo en enfermedades nefrológicas como: la insuficiencia renal aguda y crónica, la diálisis peritoneal y la hemodiálisis, las enfermedades glomerulares y la hipertensión renovascular, cubre también otras patologias y temas urológicos o nefro-urológicos como: el trasplante renal, las infecciones urinarias y la nefrolitiasis. Ha publicado hasta el momento algunas revisiones sobre el tratamiento y la prevención de las infecciones urinarias en niños y adultos ${ }^{7}$.

El Grupo Cochrane de Trastornos Menstruales y Subfertilidad. Más orientado a transtornos ginecológicos incluye también el estudio de la subfertilidad así como revisiones sobre el papel de la cirugía o embolización del varicocele en varones subfértiles, o sobre las técnicas quirúrgicas para la recuperación de espermatozioides para ICSI en azospermia ${ }^{8}$.

Otros grupos no directamente relacionados con la especialidad pueden incluir revisiones de interés para el urólogo como las revisión del Grupo Cochrane de Dolor, Apoyo y Curas Paliativas sobre la dipirona en el tratamiento del dolor cólico renal agudo o sobre la radioterapia o los bifosfonatos en el tratamiento del dolor por metástasis óseas ${ }^{9}$.

Además de la elaboración de revisiones sistemáticas, la Colaboración Cochrane aporta, a la medicina en general y a la urología en particular, una excepcional herramienta de información como es el Registro Central Cochrane de Ensayos Controlados (The Cochrane Central Controlled Trials Register-CCTR), que permite localizar todos los ensayos clínicos controlados sobre cualquier tema de interés. 


\section{CONCLUSIONES}

La Biblioteca Cochrane es una excelente herramienta para la búsqueda de información. Permite localizar tanto estudios primarios, ensayos clínicos controlados, a través de su Registro Central Cochrane de Ensayos Clínicos, como secundarios, revisiones sistemáticas elaboradas por la Colaboración Cochrane y por otras instituciones.

Los trabajos de la Colaboración Cochrane en el campo de la urología, existentes hasta el momento, aportan ya abundante información, resumida y sistematizada sobre el tratamiento de distintas patologías con elevada prevalencia como la hiperplasia prostática benigna, el cáncer de próstata, la incontinencia urinaria o las infecciones urinarias.

\section{REFERENCIAS}

1. Cochrane AL. 1931-1971: a critical review, with particular reference to the medical profession. In: Medicines for the year 2000. London: Office of Health Economics, 1979, 1-11.

2. Tríptico de difusión de la Colaboración Cochrane. http://www.cochrane.es/Download.
3. La Red Cochrane Iberoamericana. http://www.cochrane.es/ Castellano/CC_Resumen.

4. Clarke M, Oxman $\mathrm{AD}$, editores. Cochrane Reviewers' Handbook 4.1 [updated June 2000]. En: Review Manager (RevMan) [Computer program]. Versión 4.1. Oxford, England: The Cochrane Collaboration, 2000. Manual de Revisores (versión española de "Cochrane Reviewers' Handbook") [actualización de Junio 2000]. Barcelona: Centro Cochrane Iberoamericano; 2001.

5. Cochrane Prostatic Diseases and Urologic Cancers Group. Abstracts of Cochrane Reviews. The Cochrane Library Issue 4, 2004 .

6. Cochrane Incontinence Group. Abstracts of Cochrane Reviews. The Cochrane Library Issue 4, 2004.

7. Cochrane Renal Group. Abstracts of Cochrane Reviews. The Cochrane Library Issue 4, 2004.

8. Cochrane Menstrual Disorders And Subfertility GrouP. Abstracts of Cochrane Reviews. The Cochrane Library Issue 4, 2004

9. Cochrane Pain, Palliative Care And Supportive Care Group. Abstracts of Cochrane Reviews. The Cochrane Library Issue 4, 2004

Dr. C. Tello Royloa

Avda. de Europa, 9 - 2 A

30007 Murcia

(Trabajo recibido el 16 noviembre de 2004) 